\title{
MSPAN: A Memristive Spike-Based Computing Engine With Adaptive Neuron for Edge Arrhythmia Detection
}

\begin{abstract}
Jingwen Jiang', Fengshi Tian', Jinhao Liang, Ziyang Shen, Yirui Liu, Jiapei Zheng, Hui Wu, Zhiyuan Zhang, Chaoming Fang, Yifan Zhao, Jiahe Shi, Xiaoyong Xue* and Xiaoyang Zeng
\end{abstract}

State Key Laboratory of ASIC and System, School of Microelectronics, Fudan University, Shanghai, China

In this work, a memristive spike-based computing in memory (CIM) system with adaptive neuron (MSPAN) is proposed to realize energy-efficient remote arrhythmia detection with high accuracy in edge devices by software and hardware co-design. A multi-layer deep integrative spiking neural network (DiSNN) is first designed with an accuracy of $93.6 \%$ in 4-class ECG classification tasks. Then a memristor-based CIM architecture and the corresponding mapping method are proposed to deploy the DiSNN. By evaluation, the overall system achieves an accuracy of over $92.25 \%$ on the MIT-BIH dataset while the area is $3.438 \mathrm{~mm}^{2}$ and the power consumption is $0.178 \mu \mathrm{J}$ per heartbeat at a clock frequency of $500 \mathrm{MHz}$. These results reveal that the proposed MSPAN system is promising for arrhythmia detection in edge devices.

Keywords: spike-based, neuromorphic computing, memristive, computation in memory, arrhythmia detection

\section{INTRODUCTION}

Recently, remote healthcare monitoring has received increasing attention for biomedical applications in edge devices. Driven by the increasing performance of artificial intelligence (AI), especially deep learning (DL), the healthcare monitoring applications has spread to various aspects including early warning, diagnosis, treatment, and prognosis (Sodhro et al., 2018; Alam et al., 2019; Patan et al., 2020; Pustokhina et al., 2020). Because such applications are usually deployed in edge devices where computing and memory resources are extremely limited, energy-efficient DL systems are highly required with corresponding software and hardware implementations.

One of the most valuable edge biomedical applications is remote monitoring of cardiovascular disease, which has become one of the most serious threats of human health nowadays (Wilkins et al., 2017; Zhou et al., 2018; Mehra et al., 2020). Evidence shows that the occurrence of cardiac accidents can be predicted by interpreting the ECG signal in advance, so as to provide valuable time for the intervention of emergency means (Ince et al., 2009; Purushothaman et al., 2014; Vafaie et al., 2014; Rajpurkar et al., 2017; Zhang et al., 2018; Attia et al., 2019; Hannun et al., 2019). Compared with the bedside ECG monitoring devices, the wearable ones are more suitable for remote longterm and real-time monitoring owing to convenient setup, thus attracting extensive research. In the scene of home monitoring, the monitoring device needs to collect the ECG signal constantly without medical staff at side (Clark et al., 2018; Ozkan et al., 2019). Therefore, the main challenge to design such systems is to make the detection of abnormal ECG signals automatic, low-power, and real-time (Yasin et al., 2017; Ai et al., 2018). 
The biologically inspired spiking neural network (SNN) has proven to be powerful in computing with low hardware costs, providing a promising solution to the challenges mentioned above. However, the existing SNN training algorithms such as tempotron (Iyer and Chua, 2020), spiking-time dependent plasticity (STDP) (Pu and Cook, 2015), remote supervised method (ReSuMe) (Ponulak and Kasiński, 2010), and SpikeProp (Bohte et al., 2000) suffered from remarkable computation cost and performance loss compared with deep neural networks (DNNs). To achieve a balance between the computation cost and the training complexity, a spiking convolutional neural network (Spiking-CNN) was proposed (Tian et al., 2021) to deal with EEG signals. A traditional CNN was first trained and then transformed to SNN with the trained weights. However, Spiking-CNN separated the training and the inferring procedures of SNN, and this may bring an uncertain degradation of the network performance. To design a spike-based training method, $\mathrm{Wu}$ et al. (2018) proposed the back propagation for spiking networks in both spatial and temporal domain, but this method failed to improve both the performance and the energy efficiency. Therefore, novel techniques using intrinsically spiking-based algorithms are still pursued to develop a hardware-friendly and high-performance SNN.

Recently, the progress of resistive random-access memory (RRAM), which is a two-terminal device applying memristors to realize resistive switching, provides potentials for energyefficient neural network deployment (Guo et al., 2019). Due to the energy-efficient features of memristors, attempts utilizing memristors to build synapses (Wang et al., 2017) and neurons (Wang et al., 2018) have been made, and achieved great progress. The conductance of RRAM can be modulated by electrical pulses either through a variably conductive filament or through the migration of oxygen vacancies (Milo, 2020; Xiao et al., 2020). In addition, RRAM has attractive features, such as high scalability, low consumption power, fast write/read speed, stable storage, and multi-value tune ability. Moreover, RRAM can be applied to inmemory computing for neural network deployment and provides appreciable potential to break the memory wall encountered by conventional von-Neumann architecture.

Multiplication and accumulation (MAC) operation, which is the major computation type in neural network processing, usually dominates the energy consumption and the latency in a hardware system. Computing in memory (CIM) embeds the MAC calculation in memory array (Verma et al., 2019) with a mixed-signal computing paradigm, which is promising to address the computational energy and performance bottlenecks encountered by conventional von-Neumann architecture. Besides, the MAC operations can be performed with high parallelism. The input vector activates multiple rows at a time and the dot product is the sum of column currents weighted by the conductance of memory cells (Strukov et al., 2009; Hu et al., 2017). However, large analog-to-digital converters (ADCs) are always required at the side of the array, which may bring huge overheads in area and energy.

Memristor has shown excellent performance in simulating both spike-based neurons and synapses in hardware, and the use of SNN is expected to further reduce the computational power consumption. However, there have been few studies on RRAM-based SNN. Compared with RRAM-based artificial neural network (ANN), the RRAM-based SNN proposed by Tang et al. (2015) requires only $1 / 7$ power consumption with a slight accuracy degradation ( $\sim 2 \%)$. However, it fails to consider the non-ideal circuit conditions such as interconnection effects and non-linear effects, and the coding mechanism of SNN also has not been properly explored. For the first time (Valentian et al., 2019), integrated the spike neural network by combining analog neurons and RRAM-based synapses to implement a perceptron design. However, the simple hardware structure can only accommodate a single fully connected layer, which has a poor classification accuracy of $84 \%$ on MNIST. Zhang et al. (2020) implemented a temporal coding SNN to recognize the Olivetti face patterns and achieved a better energy efficiency. However, the hardware scale is too small to store enough weights, making it difficult to accomplish complex tasks. To sum up, the works mentioned above are all based on the traditional image recognition database and may not perform well for healthcare applications like arrhythmia detection.

In this work, to overcome the above-mentioned problems, a memristive spike-based computing engine with adaptive neuron - MSPAN is proposed by software and hardware codesign to realize an energy-efficient approach for biomedical application in edge devices. Our contributions lie in the following aspects:

(1) An energy-efficient deep integrative spiking neural network (DiSNN) is proposed as well as the training and inferring strategies. The computation complexity can be largely reduced compared to CNN-based methods while keeping high performance.

(2) A memristor based ADC-free CIM architecture is proposed for inference with threshold adaptive leaky integrate and fire (LIF) neuron module to mimic the function of human brains.

(3) The 4-bit signed mapping method and the corresponding weighted current mirror addition (WCMA) circuit are proposed for the proposed DiSNN to further reduce the area and power consumption overheads.

(4) The proposed memristive system is applied to dealing with biomedical signals in edge devices and achieves both high accuracy and energy efficiency in ECG-based arrhythmia detection tasks.

The remaining sections of this paper are organized as following. Section "Materials and Methods" introduces the key theories and modules of the proposed system. Experimental results and discussions are described in section "Results and Discussion." Section "Conclusion” concludes this paper.

\section{MATERIALS AND METHODS}

In this section, the key modules and the corresponding theories of the proposed MSPAN are described in detail, including the spike encoder design, adaptive LIF neuron model, the structure of DiSNN as well as its computing strategy, the memristor based 
CIM architecture design, the memristor based neural dendrites, the signed weight mapping scheme with corresponding circuits and the threshold-adaptive neuron circuit design.

\section{Poisson Spike Encoder Design}

A spike encoder transforms the input data to the spike sequences that can be processed by SNNs. In this study, a Poisson spike encoder is designed based on the Poisson random number generation. The encoding process can be presented as

$$
\delta_{i}= \begin{cases}1, & X_{i}<I_{i} \\ 0, & X_{i}=I_{i}\end{cases}
$$

where the subscription $i$ stands for the $\mathrm{i}$-th element in tensor I and $\mathrm{X}$. I is the input tensor, and $\mathrm{X}$ is a randomly generated tensor, whose values of elements distributes uniformly in the range of $(0,1)$. For each time step $t$ in the time window $T$, the encoder would generate a spike tensor $\delta_{t}$. According to the definition of Poisson distribution, the total number of spikes at one position in the whole time window would follow Poisson distribution approximately when time window $T$ is large enough. That is the reason why this encoder was named Poisson encoder.

The mean value of spikes generated at position $i$ can be obtained through

$$
\lambda=T I_{i}
$$

\section{Adaptive Leaky Integrate and Fire Neuron Model}

Leaky Integrate and Fire model is often used to emulate neuronal behaviors (Lapique, 1907). Inspired by natural behavior patterns of biological neurons, the proposed adaptive LIF neuron model in this work can avoid from being constantly active or inactive. Otherwise, the network performance may be hugely degraded. Besides, a self-modulated function is employed in this work to ensure that the pre-synaptic stimulus of each neuron be constantly non-negative, thus making the model more biological plausible and perform better. Mathematically, the self-modulated function can be expressed by Eqs 3-7 as below:

$$
\begin{gathered}
\tau \frac{d u}{d t}=-u+I R_{0}, \quad u<V_{t h} \\
u=0, \quad u \geq V_{t h} \\
o= \begin{cases}1, & u \geq V_{t h} \\
0, & u<V_{t h}\end{cases} \\
I_{0_{L I F}=\sum_{j=1}^{l} w_{j} o_{j}^{p r e v}} \\
I= \begin{cases}I_{0_{L I F}} & I_{0_{L I F}} \geq 0 \\
0, & I_{0_{L I F}}<0\end{cases}
\end{gathered}
$$

where $u$ denotes the membrane potential of the neuron, $R_{0}$ the unit resistance (equals 1 in value), $\tau$ the time constant, $I_{0_{L I F}}$ the weighted sum of all the spike inputs which stand for original presynaptic stimuli from the previous layer at the current time step, $l$ the length of the last layer, $I$ the modified pre-synaptic stimulus, and $o, o^{\text {prev }}$ the output spike of the current layer and the previous layer, respectively. $V_{t h}$ is the threshold voltage that is adaptive for neurons in every layer of the proposed SNN structure, as discussed in the next section. Whenever the membrane potential of a neuron exceeds the threshold voltage, it will generate a spike, and then reset its membrane potential to the reset voltage, as shown in Figure 1.

The equations above give the differential form of the function of a LIF neuron model. To train the network iteratively, the model needs to be transformed into an iterative form. From Eq. 3, using Euler method, we can obtain that

$$
u_{t+1}=\left(1-\frac{d t}{\tau}\right) u_{t}+I R_{0}
$$

where $u_{t 1}-u_{t}=d u$, and the subscript denotes the time step $t$. Define

$$
k_{\tau}=1-\frac{d t}{\tau}
$$

as the decay constant, and the iterative form can be expressed as below:

$$
u_{t+1}=k_{\tau} u_{t}+I R_{0} .
$$

\section{Deep Integrative Spiking Neural Network}

Figure 2B gives the model structure of the proposed DiSNN topology. The DiSNN topology consists of an input layer, five spike-based convolution (SConv) layers, two spike-based fully connected (FC) layers and an output layer. Two max-pooling layers are inserted between the SConv layer and the following one in the second and the fifth layers, respectively. To provide convenience for hardware design, single-dimension (1-D) kernels are employed to extract features from the raw ECG data.

Figure 2A shows the computing strategy of DiSNN. The features of the input ECG sample are interpreted as spike sequences at each time step by the spike encoder. Each SConv or FC block of DiSNN has an integrate unit (IU) and an adaptive unit (AU). The IU integrates the information from the former block while the AU adapts the information to extract the true features against the noise. The update of membrane potential in the LIF neuron is realized jointly by IU and AU at every time step. Figure 3 shows the weight distribution of the five SConv blocks of DiSNN, which is expressed in the format of SConv - number of the block, output channel $\times$ input channel $\times$ kernel height $\times$ kernel width.

At every time step, the output neurons of DiSNN may be activated as needed. A spike counter records the activation number of all the output neurons over all time steps and the one with the highest average activating frequency tells the results of classification. All the data transferred between DiSNN blocks is in single-bit ( 0 or 1 ) form, which only requires accumulation operations to accomplish the computing process. The elimination of multiplication operations can significantly reduce computation complexity and potential hardware costs. Therefore, the proposed DiSNN is more hardware-friendly, promising for low-cost edge applications. 


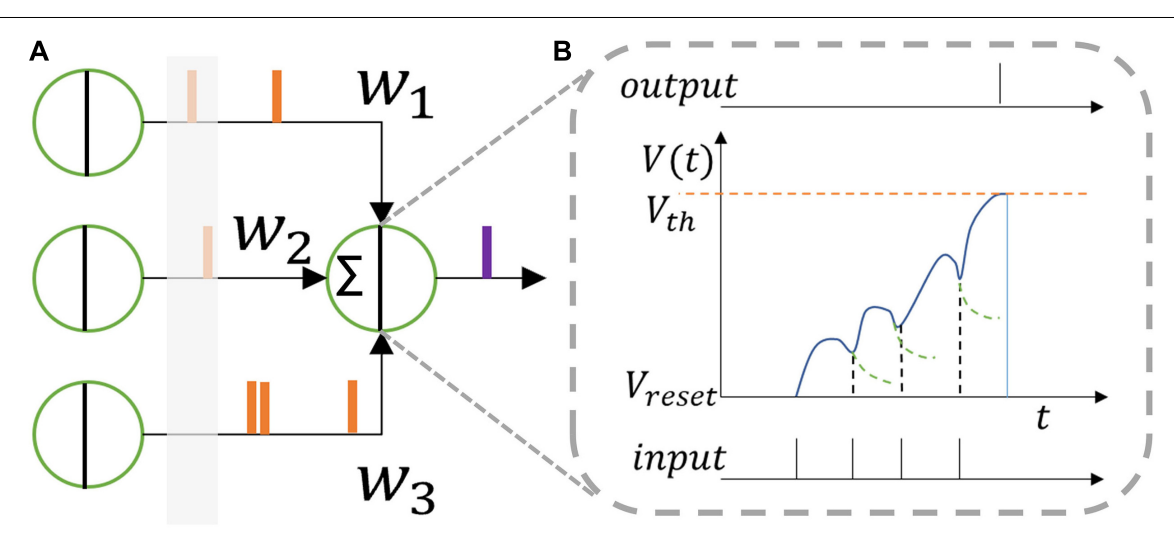

FIGURE 1 | (A) Leaky integrate and fire neuron model and (B) its function. One neuron receives spikes from the former layer which are integrated inside the neuron membrane. The membrane voltage leaks when no spike comes. When the membrane voltage reaches the threshold voltage, the neuron outputs a spike to the next layer.

\section{Training and Inferring Method}

In the proposed DiSNN, the data flows simultaneously in both temporal and spatial forms. A back propagation algorithm has been given to train spiking neural networks in both domains (Wu et al., 2018). To take both time domain (TD) and spatial domain (SD) into account, the training framework is provided in the following steps.

Assuming function $\mathrm{L}$ as the loss function, the gradient descent of each layer is determined as follows. $\mathrm{L}$ is presented in Eq. 11:

$$
L=\frac{1}{2 M} \sum_{m=1}^{M}\left|y_{m}-\frac{1}{T} \sum_{t=1}^{T} o_{m, t}\right|^{2}
$$

where the subscript $m$ denotes the $m$ th sample, and $t$ the $t$ th time step. $M$ is the number of samples in one batch, and $o, y$ the output spikes and corresponding labels, respectively. By utilizing the chain rule in $n$th layer and $t$ th time step, the gradients can be obtained through Eqs 12, 13

$$
\begin{gathered}
\frac{\partial L}{\partial u_{i, t, n}}=\frac{\partial L}{\partial o_{i, t, n}} \frac{\partial o_{i, t, n}}{\partial u_{i, t, n}}+\frac{\partial L}{\partial o_{i, t+1, n}} \frac{\partial o_{i, t+1, n}}{\partial u_{i, t, n}} \\
\frac{\partial L}{\partial o_{i, t, n}}=\sum_{j=1}^{l_{n+1}} \frac{\partial L}{\partial o_{i, t, n+1}} \frac{\partial o_{j, t, n+1}}{\partial o_{i, t, n}} \frac{\partial L}{\partial o_{i, t+1, n}} \frac{\partial o_{i, t+1, n}}{\partial o_{i, t, n}}
\end{gathered}
$$

where $l_{n+1}$ denotes the total number of neurons in the $n+1$ th layer. Note that the mathematical form of a spike is a singularity function, i.e., the Dirac function, and cannot be differentiated. To make the partial difference of an output spike computable on hardware, a rectangular pulse function in Eq. 14

$$
h(u)=\left\{\begin{array}{l}
a,\left|u-V_{t h}\right| \leq \frac{a}{2} \\
0,\left|u-V_{t h}\right|>\frac{a}{2}
\end{array}\right.
$$

is used to replace the Dirac function, and the length of pulse a stands for a hyper-parameter. Applying the functions of LIF neuron models described in section "Adaptive Leaky Integrate and Fire Neuron Model" to Eqs 7, 8, the gradient of weights can be expressed in Eq. 15

$$
\frac{\partial L}{\partial w_{n}}=\sum_{t=1}^{T} \frac{\partial L}{\partial u_{t, n}} o_{t, n-1}
$$

It is worth noting the fact that a decline in accuracy might occur because of the "Spiking Stall," which means some neurons keep constantly active through all the time steps and lose the ability to send information to the next layer. In DiSNN, a hardwarefriendly method is proposed to solve this problem, i.e., the AUs.

Parallel neuron layers named auxiliary inhibitory layers are assumed for the main structure of the network as shown in Figure 2. Each of the auxiliary inhibitory layers has the same feature with the corresponding active layer and can also be trained. When updating the membrane potential, the weighted sum of all the spike inputs of the original pre-synaptic stimulus $\mathrm{I}_{0 L I F}$ is shown in Eq. 16

$$
I_{0_{L I F}}=\sum_{j=1}^{l}\left(w_{j} j_{j}^{\text {prev }}-\beta w_{j}^{a} o_{j}^{\text {prev }}\right)
$$

where $\beta$ is a hyper parameter controlling the intensity of the negative feedback. By constructing the auxiliary inhibitory layers, even if the previous layer sends out an intense stimulus, the rise of the membrane potential will remain within a reasonable range, solving the problem of "Spiking Stall."

As for the inferring process, the membrane potentials of adaptive LIF neurons of each layer is calculated at every time step and the neuron fires a spike when reaching the threshold. The output neuron accumulates the output spikes over all the time steps. The classification result is given by the index of the output neuron with the highest activation frequency on average.

\section{Computation in Memory Architecture}

The overall hardware architecture of the proposed MSPAN system for inference consists of the CIM structure and the neuromorphic circuits, as shown in Figure 4. Compared with 

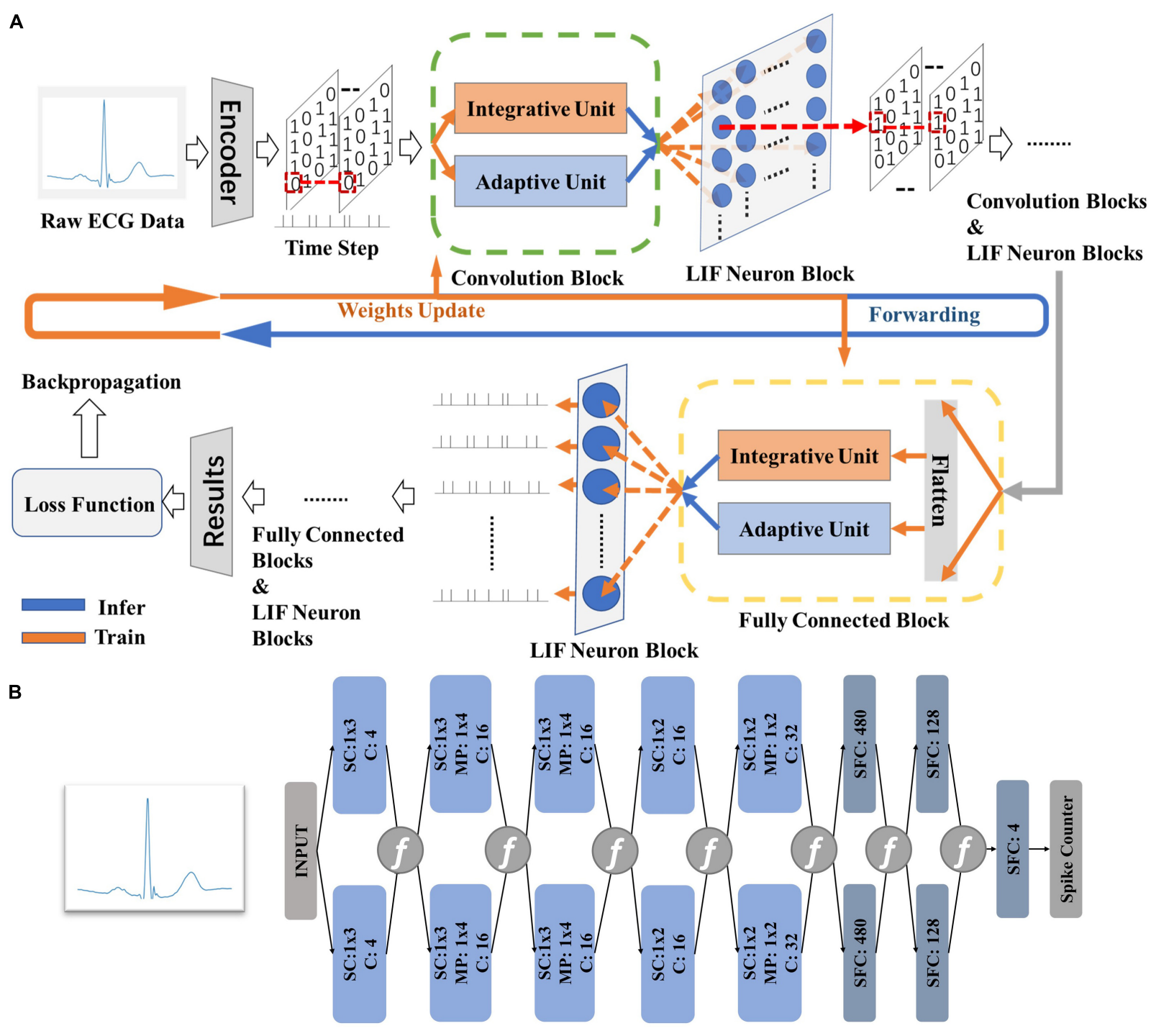

Fully Connected Block

FIGURE 2 | The theory of the proposed DiSNN. (A) The computing strategies of DiSNN; (B) the model structure of proposed in this work, where "SConv" is spike-based convolution layer, "MP" is max-pooling layer, and "C" stands for channel.

conventional architectures, CIM structure is well known to perform the MAC operations with high energy efficiency and low area consumption (Chi et al., 2016; Shafiee et al., 2016).

The inference phase of the trained DiSNN network is deployed to the above-mentioned architecture, where the 32-bit floatingpoint (FP32) synaptic weights are quantized, mapped, and loaded into 4 neighboring RRAM cell in a single row in advance. The RRAM cells acting as biological synapses receive the spike-based input data streams from the Spike Encoder based on Poisson distribution as presynaptic input spikes.

In the RRAM-based synaptic array, the word line (WL) is connected to the gate of corresponding transistor to control the on and off of the transistor in a one-transistor-one-RRAM (1T1R) cell as shown in Figure 4. The source line (SL) is connected to the source of the transistor. The upper electrode of RRAM is attached to the bit line (BL), while the lower electrode is serially attached to the drain of the transistor. According to the Kirchhoff's Law, the output of synaptic array on each BL represents the product of the input and the corresponding weight, in the form of current. During operations, six rows are selected by the WL/SL driver. The input 1-bit pulses are first applied on the SLs and then weighed by the corresponding 4 parallel $1 T 1 R$ cells. Each BL naturally adds the result of 6 dot products in the form of current according to Kirchhoff's current law (KCL). The 1-bit inputs and 1-D SConv kernels make it more efficient for network mapping and SConv operations in the proposed CIM-based system. $I_{B L}$ represents the sum of the result of 6 dot products. Two adjacent groups of BLs (a group contains 4 columns, as defined in subsequent sections) are selected by the column MUX module at one time, and then subtracted in a weighted manner, mimicking the neuromorphic impact of inhibitory neurons on active neurons. Then, the obtained 

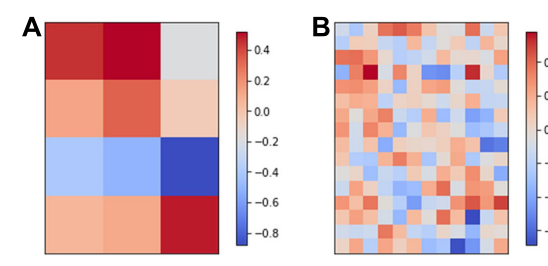

D

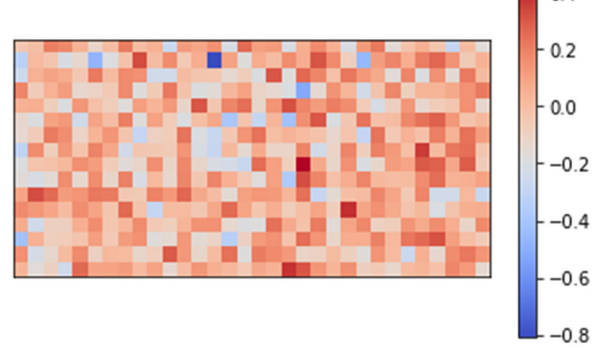

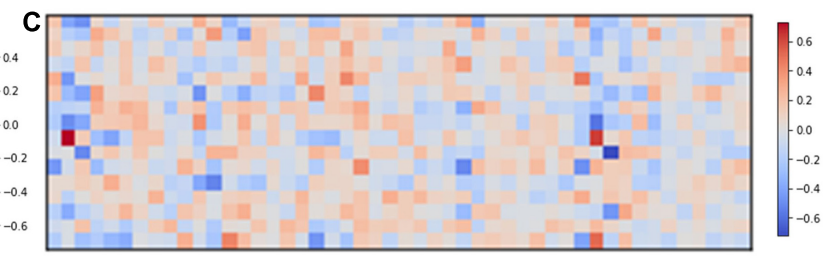

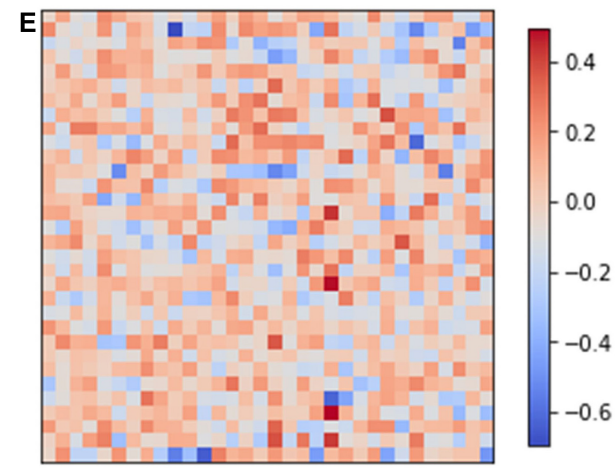

FIGURE 3 | Weights of the five spike-based convolution blocks. (A) SConv1, $4^{*} 1^{*} 1^{\star} 3$; (B) SConv2, 16*4*1*3; (C) SConv3, 16*16*1*3; (D) SConv4, 16*16*1*2; and (E) SConv5, 32*16*1*2.

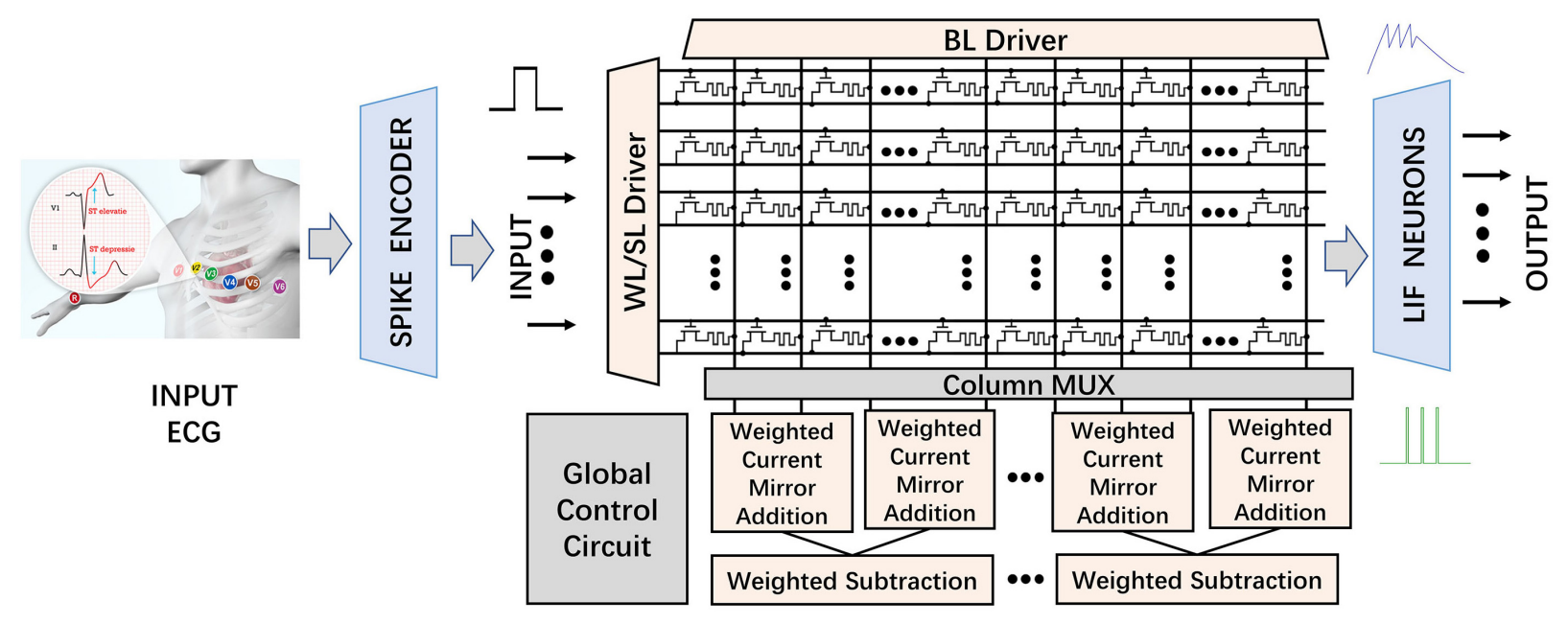

FIGURE 4 | Structure of the proposed MSPAN system ("WL" stands for word line; "SL" stands for source line; "BL" stands for bit line; and "MUX" stands for multiplexer).

pre-synaptic stimulus currents are applied to the corresponding threshold-adaptive LIF neurons, whose membrane will sample and integrate the input pulses non-linearly and then fire a spike once the accumulated voltage surpasses $\mathrm{V}_{\text {threshold }}$. A set of latches would record the output of each output neurons. Finally, A digital counter adds up the number of pulses emitted by the output LIF neurons through all the time steps and then the classification result is determined by the neuron with the highest activating frequency on average.

\section{Memristor Based Neural Dendrites}

The typical scheme for weight storage (Chi et al., 2016) use two memory arrays to separately store the positive and negative weights of neural networks, as shown in Figure 5. The matrix multiplication operations are first performed separately in each array and then the intermediate results are subtracted in peripheral circuits for the final results. However, this scheme leads to considerable cost in chip area and power consumption. For example, for a signed weight of $k$ bits, a total of $2 \times(k-1)$ memristors are required to represent the weight. When it comes to high-precision weights, the overhead of the memristor on the storage array will become tremendous.

In this work, we employ a sign bit to mark the polarity of weights to reduce the area and energy consumption. Besides, the weights are quantized from the FP32 precision to the 4-bit signed fixed-point one which ranges from -8 to +8 , to further reduce 

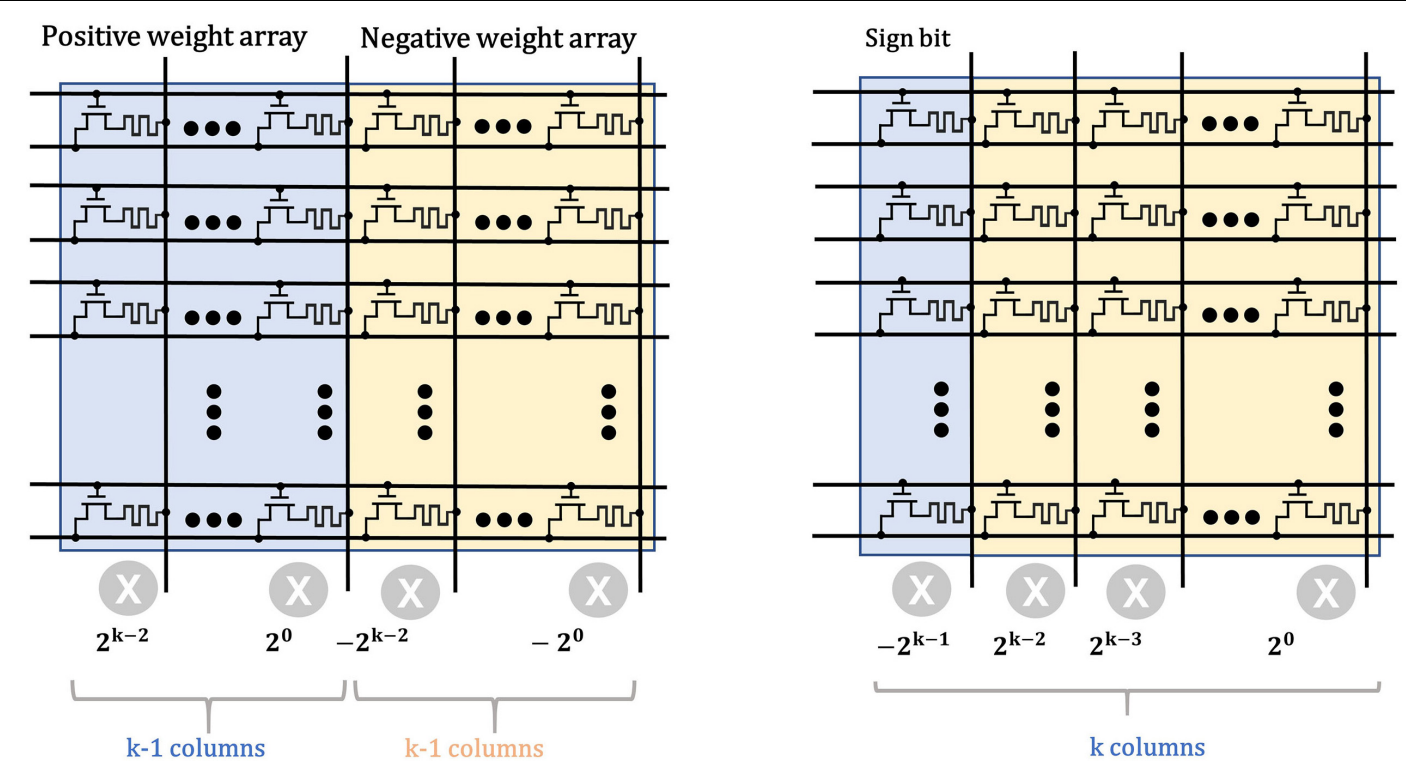

FIGURE 5 | Typical weight storage structure (left) for sign weights compared with the proposed structure (right).

the size of neural network and the overhead of weight storage. Here, the base $\left\{-2^{3}, 2^{2}, 2^{1}, 2^{0}\right\}$ is used to uniformly represent the 4-bit signed numbers and the mapping scheme is shown in Table 1. For example, the decimal $(-5)_{10}$ equals to $1 \times\left(-2^{3}\right)+$ $0 \times 2^{2}+1 \times 2^{1}+1 \times 2^{0}$, thus it is converted to $(1011)_{2}$.

The classical 1T1R cells are adopted in the proposed CIM structure to simulate the biological dendrites and store the weights in the form of the conductance of RRAM. Single level cell (SLC) RRAMs are used to implement the network weights, where the high-resistance state (HRS) of SLC RRAM is employed to represent " 0 ," and the low-resistance state (LRS) for "1," as shown in Figure 6. Since the floating-point weights have been quantized to a 4-bit signed one, four 1T1R cells are placed in parallel to store one weight in advance during write operation. The 1-bit input data is converted into different voltage levels, " 0 " for $0 \mathrm{~V}$ while "1" for $V_{\text {read }}$.

As shown in Figure 7, for SConv operation, the 1-D kernels are first split in the input channel direction, then connected and jointed into an array of slender bars, and finally stored in four corresponding parallel columns of the RRAM array. The width of

TABLE 1 | Signed 4-bit RRAM weight mapping table.

\begin{tabular}{llllcc}
\hline W[3] & W[2] & W[1] & W[0] & Binary value & Value \\
\hline $\operatorname{LRS}(1)$ & $\mathrm{HRS}(0)$ & $\mathrm{HRS}(0)$ & $\mathrm{HRS}(0)$ & 1000 & -8 \\
$\operatorname{LRS}(1)$ & $\mathrm{HRS}(0)$ & $\mathrm{HRS}(0)$ & $\operatorname{LRS}(1)$ & 1001 & -7 \\
& $\ldots$ & & & & \\
$\operatorname{LRS}(1)$ & $\operatorname{LRS}(1)$ & $\operatorname{LRS}(1)$ & $\operatorname{LRS}(1)$ & 1111 & -1 \\
$\mathrm{HRS}(0)$ & $\mathrm{HRS}(0)$ & $\mathrm{HRS}(0)$ & $\mathrm{HRS}(0)$ & 0000 & 0 \\
& $\ldots$ & & & & \\
$\mathrm{HRS}(0)$ & $\operatorname{LRS}(1)$ & $\operatorname{LRS}(1)$ & $\mathrm{HRS}(0)$ & 0110 & 6 \\
$\mathrm{HRS}(0)$ & $\operatorname{LRS}(1)$ & $\operatorname{LRS}(1)$ & $\operatorname{LRS}(1)$ & 0111 & 7
\end{tabular}

each SConv kernel is set to be 1 to better simulate the biological mechanism and reduce the computational overhead. Therefore, the size of each mapped kernel in the RRAM array is equal to $4 \times\left(K H 1 \times C_{I N} 1\right)$ which can be considered as a group. The oddnumbered groups store the weights of integrative SConv kernels (named as $I_{i}, \quad i=1,2,3, \ldots K$ ) (suppose $K$ as the number of output channels of the layer), while the adjacent even-numbered groups store the corresponding adaptive SConv kernel weights (named as $\left.A_{i}, i=1,2,3, \ldots K\right)$. Group $I_{i}$ and Group $A_{i}$ are placed closely together to facilitate the input voltage sharing scheme and the subsequent weighted subtraction operations. For multiple output channels in one SConv bank, different groups in the RRAM array store the weights of different SConv kernels in parallel, which are arranged as $I_{1}, A_{1}, I_{2}, A_{2}, \ldots, I_{K}, A_{K}$ in order. As for FC layers with no SConv kernels, the weight matrixes can be directly mapped to the synaptic array based on the proposed 4-bit signed weight mapping scheme.

\section{Bit-Wise Weighted Current Mirror Addition Circuit}

According to the proposed weight mapping scheme, the sign bit is 1 for the negative quantized weights and the corresponding RRAM is in LRS. The current $I_{L R S}$ of the sign bit needs to be multiplied by the basic weight $-2^{3}$ during bit-merging operation. If the sign bit is 0 , which indicates that the weight is positive, the current $I_{H R S}$ of the sign bit does not need to be included. The synaptic array structure implemented by the $1 \mathrm{~T} 1 \mathrm{R}$ cells makes the BL currents flow in single direction, thus it requires special processing of the BL current corresponding to the sign bit.

According to the SConv kernel size of DiSNN, the kernel height is two or three. So, we take their least common multiple, six, as the degree of parallelism. Considering that $I_{L R S} \gg I_{H R S}$ [in the simulated RRAM model (Jiang et al., 2016), the on/off ratio 


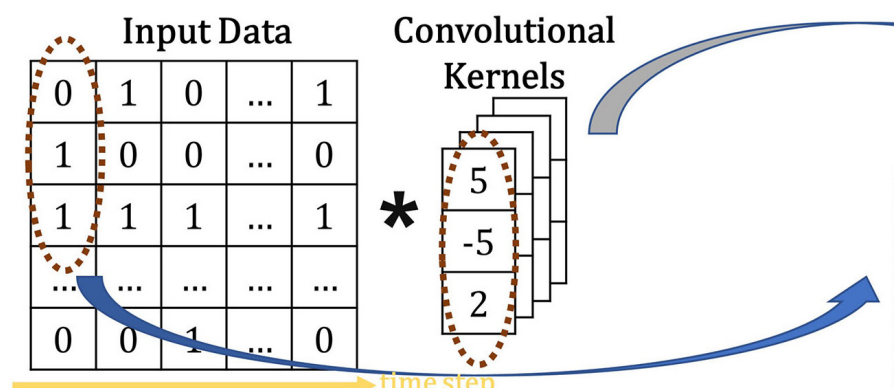

$(\mathbf{1 0 1 1})_{2}=1 *\left(-2^{3}\right)+0 * 2^{2}+1 * 2^{1}+1 * 2^{0}=(-5)_{10}$
$(0101)_{2}=0 *\left(-2^{3}\right)+1 * 2^{2}+0 * 2^{1}+1 * 2^{0}=(5)_{10}$
$(0011)_{2}=0 *\left(-2^{3}\right)+0 * 2^{2}+1 * 2^{1}+0 * 2^{0}=(2)_{10}$

$\begin{array}{l:l:l}\mathrm{V}_{1}=0 \mathrm{~V} & \times & \mathrm{W}_{1,1}=(0101)_{2} \\ \mathrm{~V}_{2}=\mathrm{V}_{\text {read }} & \times & \mathrm{W}_{1,2}=(1011)_{2} \\ \mathrm{~V}_{3}=\mathrm{V}_{\text {read }} & \times & \mathrm{W}_{1,3}=(0010)_{2}\end{array}$

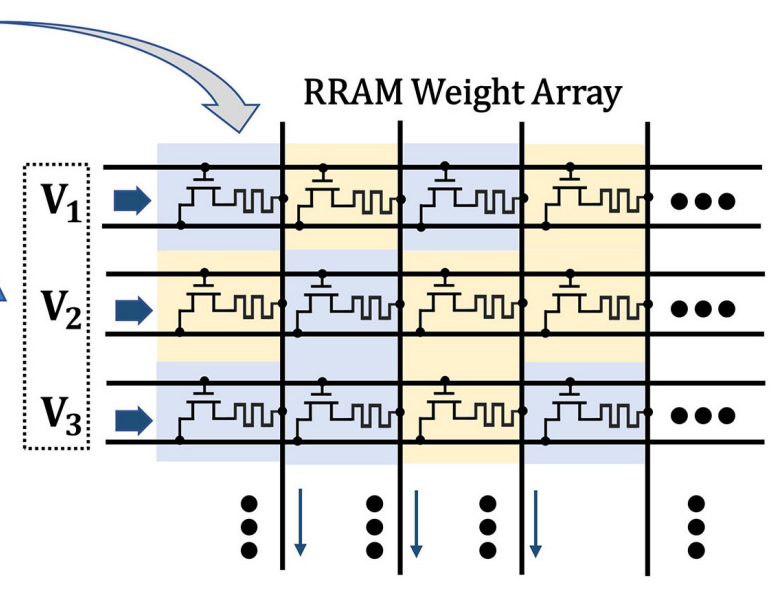

Stands for HRS
Stands for LRS

FIGURE 6 | The illustration of spike-based convolution operation with memristor crossbar, where the input data in timestep has been transformed into input voltage and the weights in SConv kernels has been transformed into RRAM resistance states.

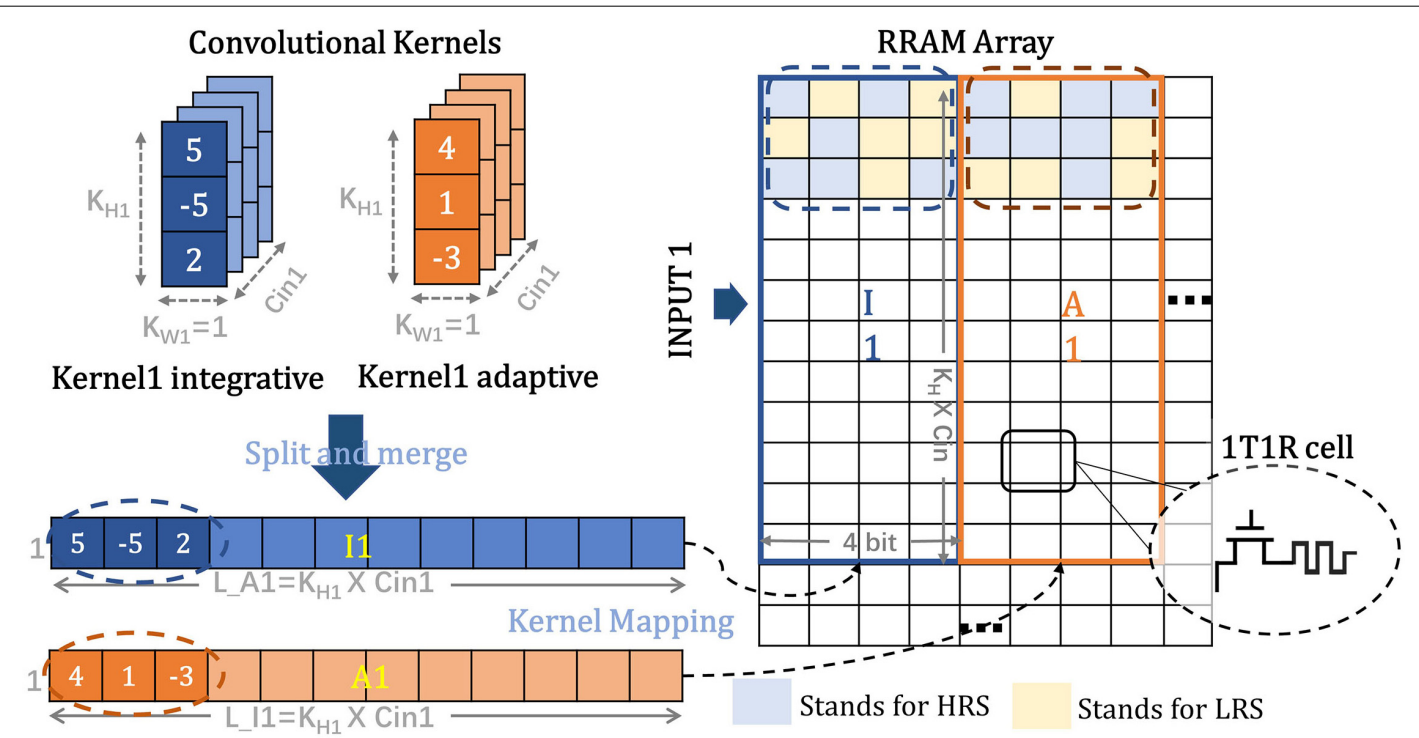

FIGURE 7 | Weight matrixes mapping method for spike-based convolutional layers where K, H, W, L, C, A, and I stand for kernel, height, width, length, channel, adaptive, and integrative, respectively.

$>100]$ and the RRAM synaptic array only processes six rows of MAC operations in parallel at a time, if there exists $I_{H R S}$ in the BL current for the sign bit, $I_{H R S}$ can be ignored compared to $I_{L R S}$. Therefore, there is no need to design different weighted addition circuits for positive and negative weights, thus greatly reducing the circuit costs.

If all of the weights processed in parallel are positive, the sign bit $I_{B L}$ [3] of six sign bits " 0 " is equal to $6 I_{H R S}$. Although the current is relatively small, when it is directly passed to the weighted current mirror circuit, it will be multiplied by the base of $-2^{3}$ and cannot be ignored, possibly impairing the accuracy of the CIM architecture to a certain extent. Here, a transmission gate is employed to judge whether the $I_{B L}$ [3] of sign bit will be connected to the WCMA circuit, as shown in Figure 8. A voltage comparator compares the voltage between $V_{r e f}$ and $V_{B L}$ formed by the sampled $I_{B L}$ through a fixed value resistor, and then produces voltage $V_{\text {TRANS }}$ connected to the gate of the NMOS in the transmission gate. If the six weights processed in parallel are all positive, the BL current of sign bit is supposed to be $6 I_{H R S}$, which is far from turning on the transmission gate, so $6 I_{H R S}$ will not be brought into subsequent calculations. However, once there exists a negative number in the six weights, then $V_{B L}$ would 


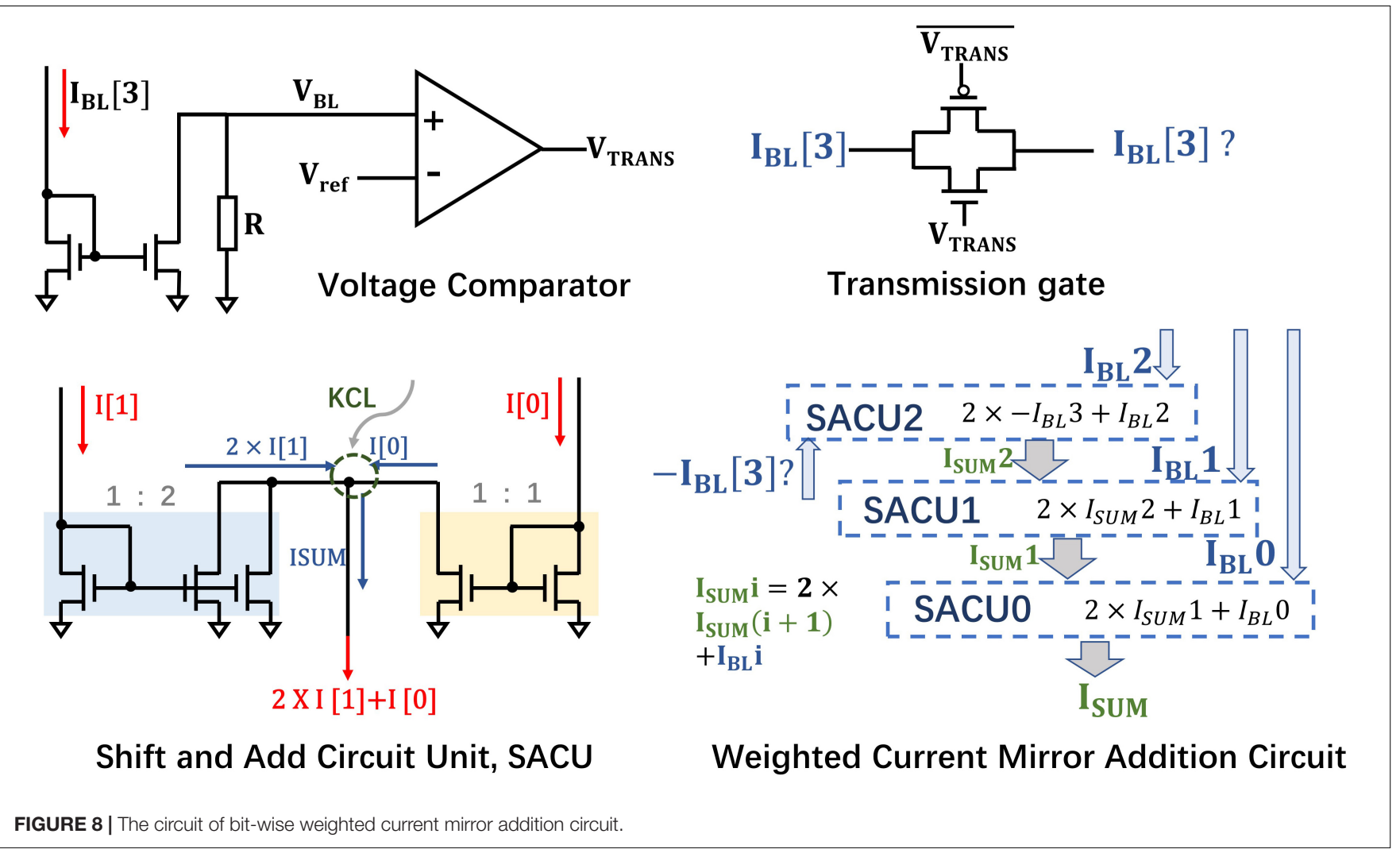

FIGURE 8 | The circuit of bit-wise weighted current mirror addition circuit.

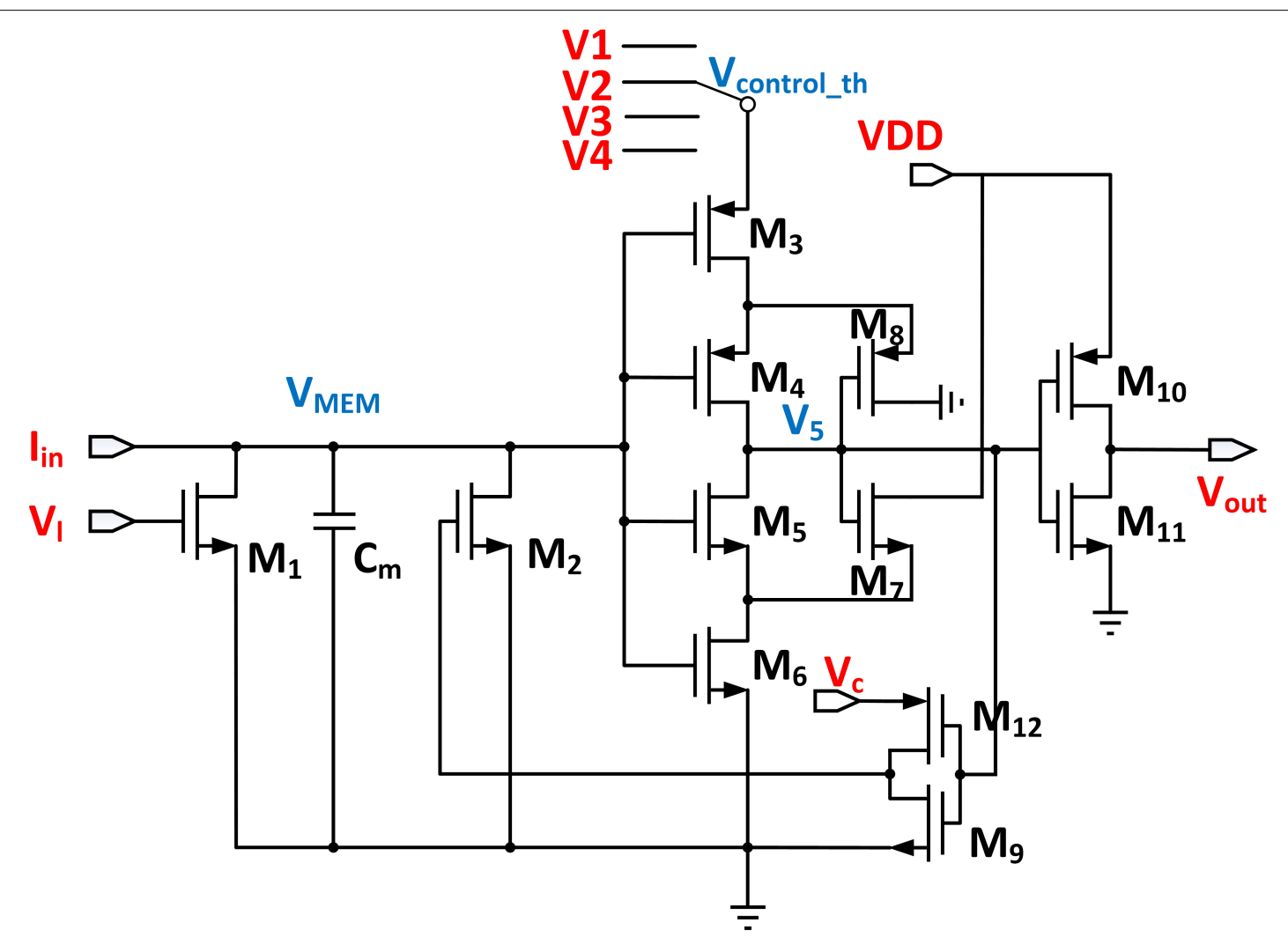

FIGURE 9 | Circuit structure of the proposed threshold-adaptive LIF neuron. 


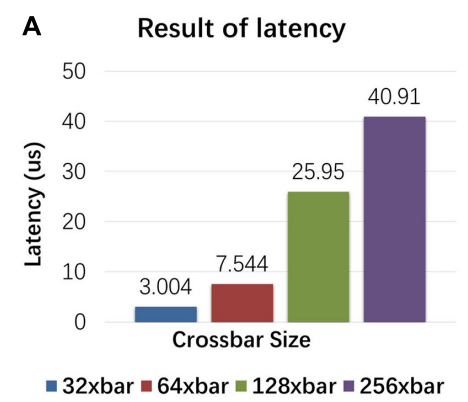

D

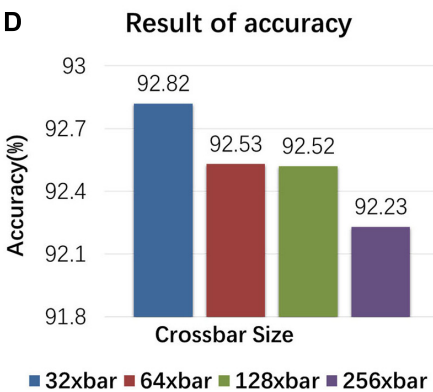

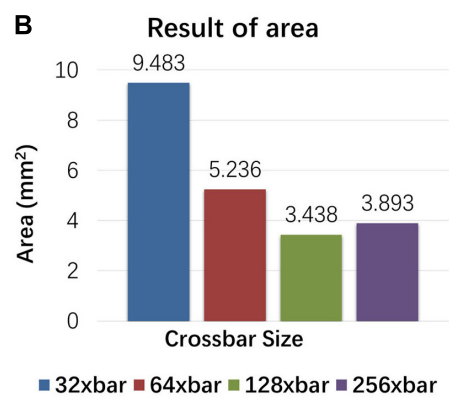

E

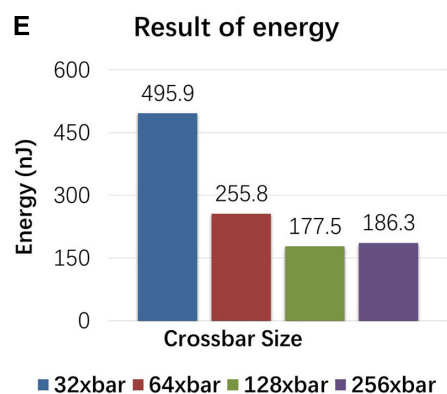

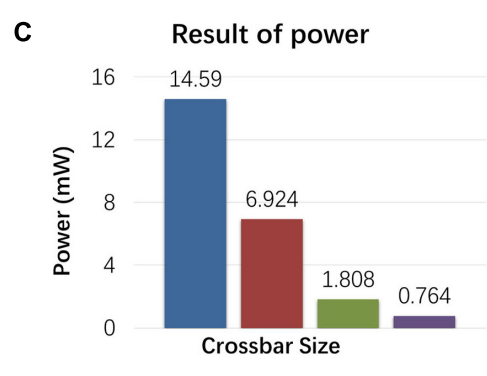

" 32xbar $\approx$ 64xbar $\approx 128 x b a r ~=256 x b a r$
F Overall hardware performance under different crossbar size

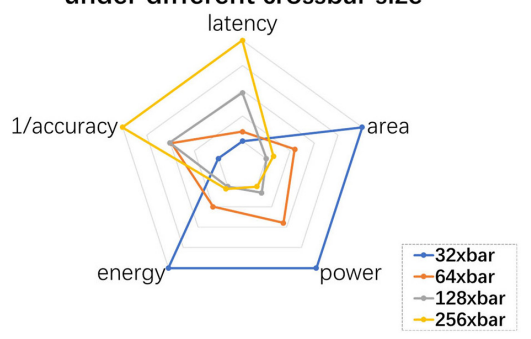

FIGURE 10 | Hardware simulation results: influence of different crossbar sizes on latency, area, power, energy, and accuracy of the proposed MSPAN system. (A) The latency of MSPAN system with different crossbar sizes; (B) the area of MSPAN system with different crossbar sizes; (C) the power of MSPAN system with different crossbar sizes; (D) the accuracy of MSPAN system with different crossbar sizes; (E) the energy of MSPAN system with different crossbar sizes; (F) radar plot of the overall hardware performance under different crossbar sizes.

be larger than $V_{\text {ref }}$ and $V_{\text {TRANS }}$ will be of high voltage, and the transmission gate is expected to open.

Furthermore, since the MAC operation satisfies the Multiplicative Distribution Law and the Associative Law, the value of each $1 T 1 \mathrm{R}$ cell can be naturally added on the BL according to KCL, and then the selected BLs will be weighted and summed. The WCMA circuit is mainly composed of 3 basic Shift and Add circuit mirror circuits (SACU). After 3 iterations, the WCMA circuit is supposed to realize the following Eq. 17:

$$
\left\{\begin{array}{rc}
I_{\text {sum }_{A_{i}}=}= & \left(-2^{3}\right) \times I_{B L_{A_{i}}}[3]+2^{2} \times I_{B L_{A_{i}}}[2]+2^{1} \times I_{B L_{A_{i}}}[1] \\
& +2^{0} \times I_{B L_{A_{i}}}[0] \\
I_{\text {sum }_{A_{i}}=}=2^{2} \times I_{B L_{A_{i}}}[2]+2^{1} \times I_{B L_{A_{i}}}[1]+2^{0} \times I_{B L_{A_{i}}}[0] & \left(I_{B L_{A_{i}}}[3]=6 I_{H R S}\right)
\end{array}\right.
$$

Equation 17 realizes the dot product of weights and input bit, in the form of analog current in Group $A_{i}(i=0,1,2, \ldots)$ (similar for integrative groups), and illustrates how the current mirror weighted addition circuit merges the currents on the four BLs. Finally, the output currents of auxiliary and inhibitory layers in the same bank will undergo a weighted subtraction operation to realize Eq. 16, mimicking the biological mechanism of lateral inhibition.

\section{Threshold-Adaptive Leaky Integrate and Fire Neuron Circuit}

The proposed threshold adaptive LIF neuron is shown in Figure 9. Since the threshold voltage of each layer in the DiSNN is specifically set, we employ a Single-Pole-Four-Throw Switch to produce different threshold voltages. Suppose that the voltage $V_{5}$ is high in the initial condition. The input current $I_{\text {in }}$ coming from the WCMA circuit will charge the capacitor $C_{m}$ to simulate the process of charge integration on the biological neuron membrane. The transistor $\mathrm{M}_{1}$ plays the role of leakage resistance, which discharges $C_{m}$ slowly and its leakage rate is decided by $V_{l}$. As the input current continues to flow in, the voltage across the capacitor continues to rise non-linearly. Once $V_{m}$ surpasses the threshold voltage that is decided by transistors M3, M4, M5, and M6, both M5 and M6 will be turned on and pull down the voltage $V_{5}$. The inverter composed of M10 and M11 then converts V5 to a high-level voltage at the output port. To reset the LIF circuit, V5 is fed back to the input port through the transistor M2, so that the integration capacitor $C_{m}$ is discharged quickly, and the discharge rate is controlled by $V_{c}$ (Yang et al., 2020). A spike will be fired before the neuron is reset. Finally, the LIF neuron enters the refractory period.

\section{RESULTS AND DISCUSSION}

\section{Evaluation of Network Performance}

To evaluate the performance of the proposed DiSNN, the MITBIH dataset is used in this work (Atzori et al., 2014). The MIT$\mathrm{BIH}$ dataset is separated into the training set and the testing set randomly with the ratio of $4: 1$. In order to train or infer with the proposed DiSNN, the input data need to be converted into the time dependent spike sequences. The input size of ECG samples 
during training is $1 \times 251$ with a batch size of 25 , which is minmax normalized into the range of $[0,1]$. The corresponding spike sequences are generated using the spike encoder.

In this experiment, when the time step of spike-encoder is set to 25 , the accuracy of the proposed DiSNN reaches 80,90 , and $95 \%$ at the 9 th, the 32 th, and the 75 th epoch, respectively. After training 100 epoches, the proposed DiSNN achieves an accuracy of $93.6 \%$ while the computation complexity is reduced by over $92 \%$ with only a decline of $4 \%$ in accuracy compared to the CNN topology of the same structure, as calculated in Eqs 18, 19.

$$
\begin{aligned}
T_{C N N}= & \sum M_{H} M_{W}\left(K_{H} K_{W}+K_{H}+K_{W}-1\right) C_{i n} C_{\text {out }} \\
& \times \text { Ops } \times \text { bit }+\sum N_{\text {in }} N_{\text {out }} \times \text { Ops } \times \text { bits } \\
T_{S C N N}= & \sum M_{H} M_{W}\left(K_{H}+K_{W}-1\right) C_{\text {in }} C_{\text {out }} \times \text { Ops } \times \text { bit } \\
& \times t+\sum N_{\text {in }} N_{\text {out }} \times \text { Ops } \times \text { bits } \times t
\end{aligned}
$$

where $T, M, N, K, C, H, W$, stand for Time Complexity, Feature Map Size, Neuron Count in FC Layers, Kernel, Channel, Height, and Width, respectively; Ops stands for the needed cycles in one computing operation ( 1 for adding, 1 for conditional branching, and 10 for multiplication); bit stands for the bit number of data flowing between two layers in $\mathrm{NN}$; $t$ stands for the number of time steps.

\section{Effect of Array Size and Memristance Fluctuation on Network Performance}

The proposed MSPAN system is evaluated on the NeuroSim platform (Peng et al., 2020). The results are evaluated at the $65 \mathrm{~nm}$ CMOS technology node and the IO bus is designed to work at a clock frequency of $500 \mathrm{MHz}$. To find out the suitable crossbar size for optimal performance, we examine the influences of crossbar size on latency, area, power, energy, and accuracy of the proposed MSPAN system, as shown in Figure 10.

As the result shows, the latency of hardware system rises as the size of the sub-array increases, which can be ascribed to the reduction of parallelism degree. The area, power, and energy also tend to decrease as the size of the sub-array increases, because the weight matrix needs to be divided into smaller sub-arrays, which results in larger overhead for additional peripheral circuits (such as WCMA circuits and LIF neurons). However, for large crossbar size, non-ideal factors such as IR drop as well as the manufacturing defects are more likely to be introduced, therefore a trade-off is needed between the hardware performance and the computation accuracy for synaptic array.

To evaluate the overall impact of array size on the hardware indicators, a radar chart is drawn intuitively in Figure 10F. The five indicators are linearly mapped to the radar chart, and the largest value of each indicator is mapped to the fifth circle. For the pentagons formed by different array sizes, the smaller the area, the better the overall performance. Therefore, under comprehensive consideration, $128 \times 128$ is selected as the crossbar size of synaptic array.

The above simulation result is based on the premise that RRAM is an ideal device, but in fact, due to the limitations of fabrication technology and unstable usage environment, RRAM devices have various non-ideal factors (Chen et al., 2017). To make the simulation result more approximate to the measurement data after taping out, the inference accuracy is re-simulated considering the conductance variation and stuck-atfaults (SAFs) problems, as shown in Figure 11.

For the conductance variation, we focus on device-to-device (d2d) variation and cycle-to-cycle (c2c) variation, which may have great impacts on the accuracy of CIM computation and the performance of DiSNN. Figure 11A demonstrates that the accuracy of the proposed hardware structure will gradually decrease as the variation (estimated as the ratio of conductance fluctuation range to the average conductance) increases, and the decline rate will increase sharply if the ratio is greater than 0.5 . Typical fabrication process and roomtemperature test environment for inference can restrict the variation parameter lower than 1.0 (Zhang et al., 2019), and the accuracy will drop by $8.49 \%$.

Stuck-at-fault is another common problem that affects the performance of RRAM crossbar chip. It is mainly caused by the defects introduced in the manufacture process, which will result in a certain percentage of RRAM cells to remain in HRS or LRS, i.e., SAF. The device in fault may be randomly distributed in the crossbar structure or assembled in the form of a whole row or a whole column. Figure 11B shows the accuracy with increasing percentage of RRAM cells of SAF. Previous work (Chen et al., 2017) claimed that, the stuck at LRS and stuck at HRS problems are supposed to affect 1.75 and $9.04 \%$ of RRAM cells in crossbar array, respectively. Based on such SAF level, the hardware accuracy rate is only reduced by $4.25 \%$, and the entire system still keeps a high performance.

\section{Simulation With Different Proportions of Neuron Configuration}

The LIF circuit in Figure 9 is simulated by Cadence Virtuoso. All transistors in the circuit except the two inverters are supposed to operate in the sub-threshold region. In the simulation process, $\mathrm{Cm}$ is set to be $0.9 \mathrm{pF}$, the $\mathrm{W} / \mathrm{L}$ ratio of PMOS is $220 / 100 \mathrm{~nm}$, the $\mathrm{W} / \mathrm{L}$ ratio of NMOS is $120 / 100 \mathrm{~nm}$, and $V D D, V_{c}, V_{I}, V 1, V 2, V 3$, and $V 4$ are set to be $1 \mathrm{~V}, 850 \mathrm{mV}, 300 \mathrm{mV}, 900 \mathrm{mV}, 950 \mathrm{mV}, 1 \mathrm{~V}$, and $1.05 \mathrm{~V}$, respectively. The threshold voltage of the proposed LIF neuron can be adjusted to accommodate the hyperparameter in the DiSNN algorithm.

Figure 12 shows the simulation waveform of the proposed threshold-adaptive LIF neuron. The three groups of graphs in Figure 12 correspond to different input conditions. In each group, the top line is the input waveform, the middle line is the potential difference across capacitor $\mathrm{Cm}$, i.e., the neuron's membrane accumulative voltage, and the bottom line is the waveform of the output pulse changing with time. The simulation results indicate that with the increase of input current amplitude, frequency, and duration, the time interval between the emitted pulses of the proposed LIF neuron becomes smaller while the frequency of the emitted pulses also increases. 

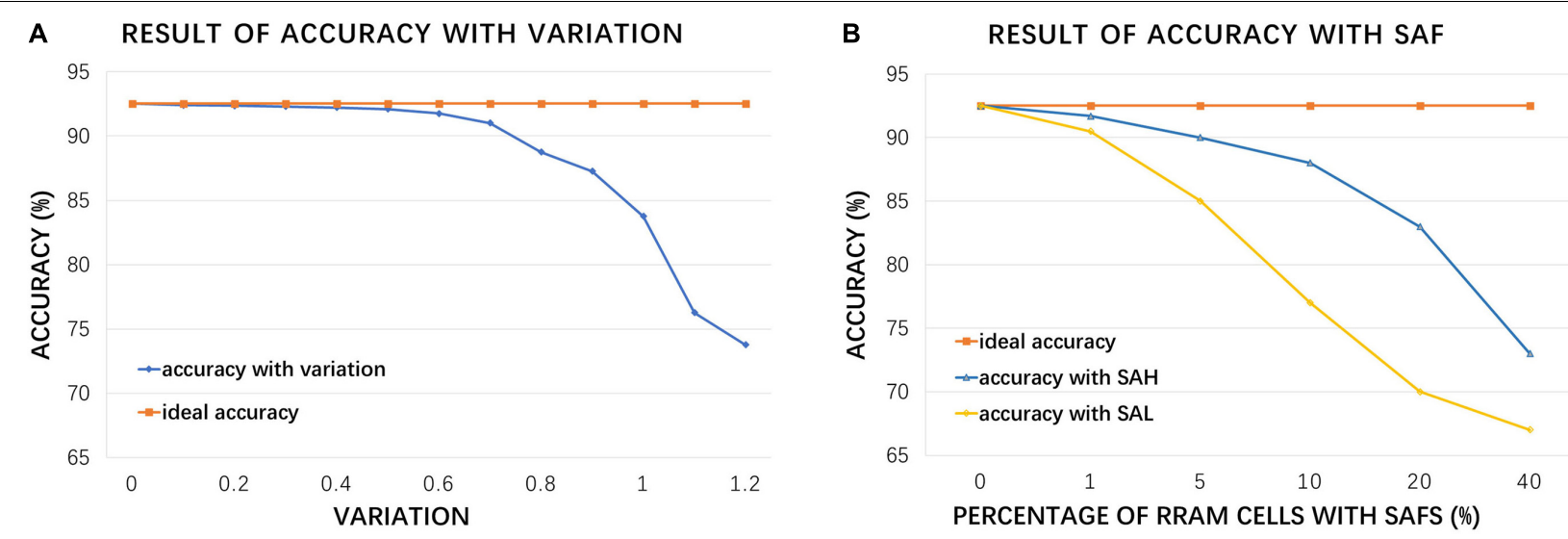

FIGURE 11 | Hardware simulation results: (A) accuracy under increasing RRAM device variation compared with ideal accuracy; (B) accuracy with increasing RRAM stuck at fault (SAF) rate, where SAH represents stuck at HRS and SAL represents stuck at LRS.
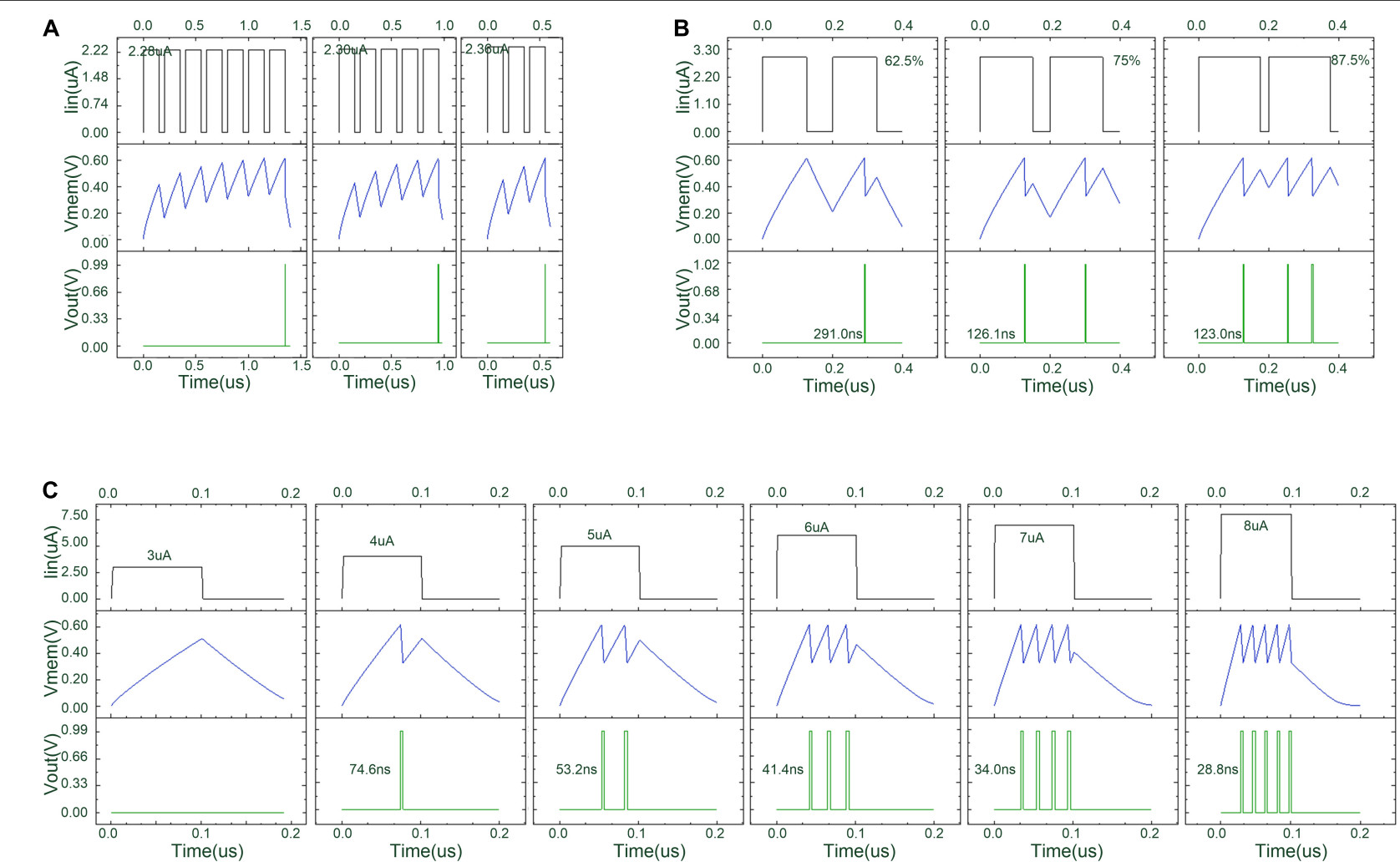

FIGURE 12 | Simulation result of the proposed threshold-adaptive LIF neuron under different input condition. (A) The emission time of a LIF neuron under a series of square waves with slightly different amplitudes; (B) the output of a LIF neuron under a series of square waves with the same amplitude but increasing duty cycles; and (C) the emitted pulse number of a LIF neuron under a series of square waves with increasing amplitude at equal intervals.

\section{Implementation Overheads}

For AI based edge systems, a tradeoff exists between the energy and the computation accuracy. As shown in Figure 13, the SNN-based systems show overwhelming advantages in energy consumption compared to CNN/ANN-based work while maintaining relatively high accuracy. Further optimization can be achieved by deploying SNN in CIM architecture. The proposed
MSPAN system deploying a 7-layer network achieves the lowest energy consumption compared with all counterparts using $65 \mathrm{~nm}$ technology.

To demonstrate the contributions of this work, the evaluation metrics including area, energy consumption, and detection accuracy are estimated and compared with other state-of-the-art work, as shown in Table 2. The proposed system exhibits at 


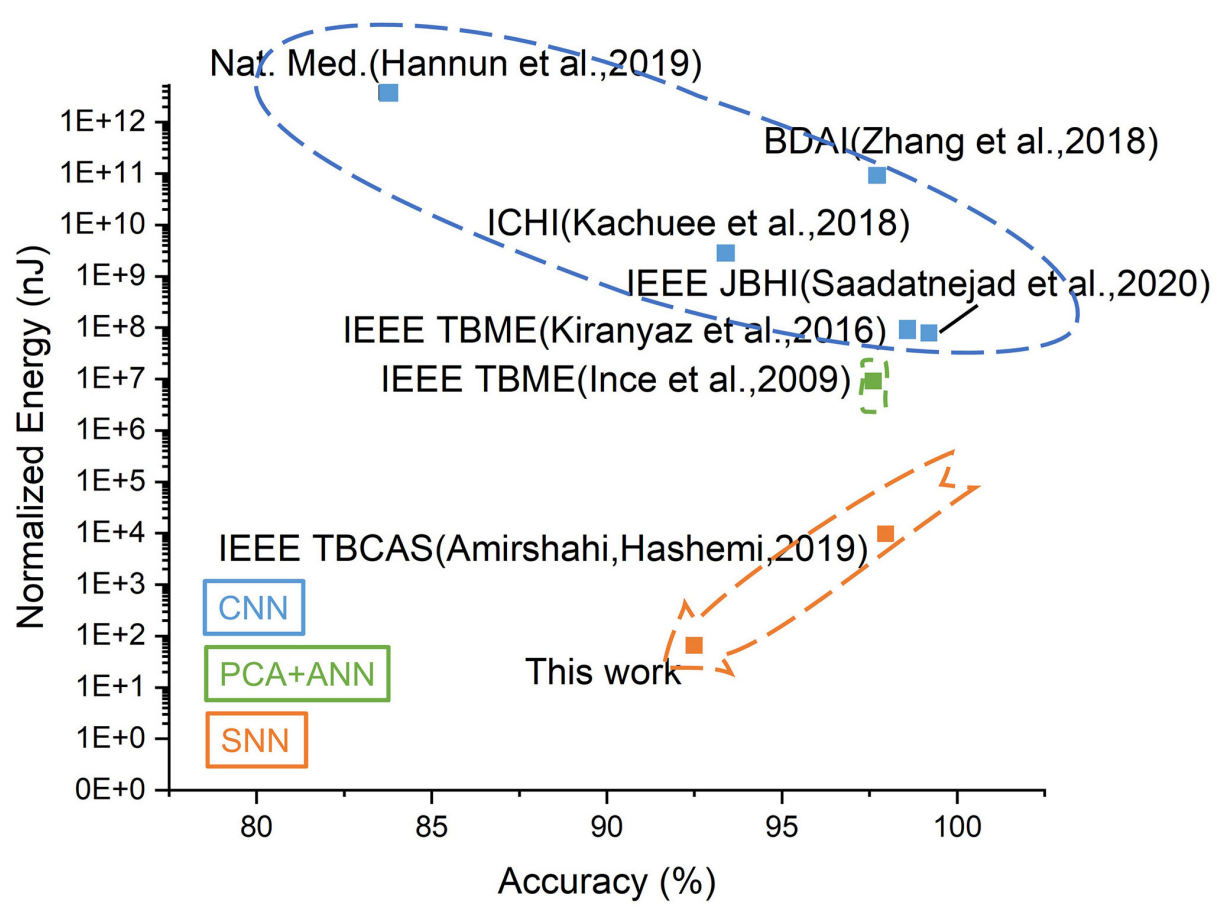

FIGURE 13 | The tradeoff between accuracy and energy for related ECG detection works. The energy has been normalized to $65 \mathrm{~nm}$ for a fair comparison.

TABLE 2 | Overall comparison with other state-of-the-art works.

\begin{tabular}{|c|c|c|c|c|c|c|c|c|}
\hline References & $\begin{array}{l}\text { Technology } \\
(\mathbf{n m})\end{array}$ & Area $\left(\mathrm{mm}^{2}\right)$ & $\begin{array}{c}\text { Energy } \\
\text { (/heartbeat) }\end{array}$ & Task type & $\begin{array}{c}\text { Detection } \\
\text { accuracy }(\%)^{\star}\end{array}$ & $\begin{array}{l}\text { Network } \\
\text { type }\end{array}$ & $\begin{array}{l}\text { Computing } \\
\text { architecture }\end{array}$ & $\begin{array}{c}\text { Device } \\
\text { type }\end{array}$ \\
\hline TBME (Kiranyaz et al., 2016) & 28 & Arm cortex & $37 \mathrm{~mJ}$ & 2-class & 98.6 & CNN & Out memory & CMOS \\
\hline JHBI (Saadatnejad et al., 2020) & 28 & Arm cortex & $35 \mathrm{~mJ}$ & 4-class & 99.2 & LSTM & Out memory & CMOS \\
\hline TBCAS (Amirshahi and Hashemi, 2019) & 28 & - & $1.78 \mu \mathrm{J}$ & 4-class & 97.9 & SNN & Out memory & CMOS \\
\hline IET (Wu et al., 2020) & 55 & 4 & $1.99 \mu \mathrm{J}$ & 3-class & 97.8 & SRNN & Out memory & CMOS \\
\hline
\end{tabular}

${ }^{*}$ The detection accuracy for each work on this table is evaluated at the software level.

least $10 \times$ energy efficiency compared with other works and also achieves a satisfying accuracy of over $93 \%$ in the 4 -class ECG classification tasks.

\section{CONCLUSION}

In this work, a memristive spike-based computing engine with adaptive neuron for edge biomedical application is proposed and evaluated on ECG-based arrhythmia detection tasks. A hardware-friendly DiSNN, named DiSNN is first put forward, which can achieve an accuracy of $93.6 \%$ on MIT-BIH dataset while the computation complexity is reduced by over $92 \%$ with a merely $4 \%$ decline in accuracy compared to the CNN topology of the same structure. To deploy DiSNN in edge devices for inference, a memristor based CIM architecture is implemented. The proposed DiSNN achieves a satisfying detection accuracy of $92.25 \%$ and the average energy consumption is only $0.178 \mu \mathrm{J}$ per heartbeat at a supply voltage of $1.0 \mathrm{~V}$ and a working frequency of $500 \mathrm{MHz}$ in $65 \mathrm{~nm}$ technology. The low energy consumption greatly surpasses related works in ECG-based arrhythmia detection field.

\section{DATA AVAILABILITY STATEMENT}

Publicly available datasets were analyzed in this study. This data can be found here: https://physionet.org/content/mitdb/1.0.0/.

\section{AUTHOR CONTRIBUTIONS}

JJ and FT designed the architecture of memristive spikebased computing engine and all the relevant experiments. FT preprocessed the dataset and designed the model structure. FT and JL designed the DiSNN and conducted the software 
experiments. JJ designed the CIM architecture and the computing pattern. JJ and YL designed the LIF neuron circuit. JJ, YL, and HW conducted experiments on the neuron circuit. JJ, FT, JZ, ZS, YL, HW, ZZ, CF, YZ, and JS contributed to experiment data collection and the writing of the manuscript. XX supervised this study. All authors discussed the results.

\section{REFERENCES}

Ai, Z., Zheng, L., Qi, H., and Cui, W. (2018). "Low-power wireless wearable ECG monitoring system based on BMD101," in Proceedings of the 37th Chinese control conference (CCC), (Wuhan: Institute of Electrical and Electronics Engineers), 7374-7379. doi: 10.23919/ChiCC.2018. 8484125

Alam, M. G. R., Abedin, S. F., Moon, S. I., Talukder, A., and Hong, C. S. (2019). Healthcare IoT-based affective state mining using a deep convolutional neural network. IEEE Access 7, 75189-75202. doi: 10.1109/access.2019.2919995

Amirshahi, A., and Hashemi, M. (2019). ECG classification algorithm based on STDP and R-STDP neural networks for real-time monitoring on ultra low-power personal wearable devices. IEEE Trans. Biomed. Circuits Syst. 13, 1483-1493. doi: 10.1109/TBCAS.2019.2948920

Attia, Z. I, Noseworthy, P. A., Lopez-Jimenez, F., Asirvatham, S. J., Deshmukh, A. J., Gersh, B. J., et al. (2019). An artificial intelligence-enabled ECG algorithm for the identification of patients with atrial fibrillation during sinus rhythm: a retrospective analysis of outcome prediction. Lancet 394, 861-867. doi: 10.1016/ S0140-6736(19)31721-0

Atzori, M., Gijsberts, A., Castellini, C., Caputo, B., Mittaz Hager, A.-G., Elsig, S., et al. (2014). Data from: Electromyography Data for Non-Invasive Naturally Controlled Robotic Hand Prostheses. Sierre: Dryad. doi: 10.1038/sdata.2014.53

Bohte, S., Kok, J., and Poutré, J. (2000). “SpikeProp: backpropagation for networks of spiking neurons," in Proceedings of the 8th European Symposium on Artificial Neural Networks, (Bruges), 419-424.

Chen, L., Li, J., Chen, Y., Deng, Q., and Li, J. (2017). “Accelerator-friendly neural-network training: learning variations and defects in RRAM crossbar," in Proceedings of the Design, Automation \& Test in Europe Conference \& Exhibition (DATE), (Lausanne: Institute of Electrical and Electronics Engineers). doi: 10. 23919/DATE.2017.7926952

Chi, P., Xu, C., Zhao, J., Li, S., Zhang, T., Liu, Y., et al. (2016). Prime: a novel processing-in-memory architecture for neural network computation in rerambased main memory. Comput. Architect. News 2016, 27-39. doi: 10.1145/ 3007787.3001140

Clark, N., Sandor, E., Walden, C., Ahn, S. I., and Lu, Y. (2018). “A wearable ECG monitoring system for real-time arrhythmia detection," in Proceedings of the IEEE 61st International Midwest Symposium on Circuits and Systems (MWSCAS), (Windsor, ON: Institute of Electrical and Electronics Engineers), 787-790. doi: 10.1109/MWSCAS.2018.8624097

Guo, Y., Wu, H., Gao, B., and Qian, H. (2019). Unsupervised learning on resistive memory array based spiking neural networks. Front. Neurosci. 13:812. doi: 10.3389/fnins.2019.00812

Hannun, A. Y., Rajpurkar, P., Haghpanahi, M. G., Tison, H., Bourn, C., Turakhia, M. P., et al. (2019). Cardiologist-level arrhythmia detection and classification in ambulatory electrocardiograms using a deep neural network. Nat. Med. 25, 65-69. doi: 10.1038/s41591-018-0268-3

Hu, M., Li, H., Chen, Y., Wu, Q., Rose, G. S., and Linderman, R. W. (2017). Memristor crossbar-based neuromorphic computing system: a case study. IEEE Trans. Neural Net. Learn. Syst. 25, 1864-1878. doi: 10.1109/TNNLS.2013. 2296777

Ince, T., Kiranyaz, S., and Gabbouj, M. (2009). A generic and robust system for automated patient-specific classification of ECG signals. IEEE Trans. Biomed. Eng. 56, 1415-1426. doi: 10.1109/TBME.2009.2013934

Iyer, L. R., and Chua, Y. (2020). "Classifying neuromorphic datasets with tempotron and spike timing dependent plasticity," in Proceedings of the International Joint Conference on Neural Networks (IJCNN), (Glasgow: Glasgow Institute of Electrical and Electronics Engineers), 1-8. doi: 10.3389/fnins.2021. 608567

\section{FUNDING}

This work was supported by the National Natural Science Foundation of China (Nos. 61834009 and 61874028) and the Opening Project of Key Laboratory of Microelectronic Devices and Integrated Technology, Institute of Microelectronics, Chinese Academy of Sciences.

Jiang, Z., Yi, W., Yu, S., Lin, Y., Song, K., Karim, Z., et al. (2016). A compact model for metal-oxide resistive random access memory with experiment verification. IEEE Trans. Electron Devices 63, 1884-1892. doi: 10.1109/ted.2016.2545412

Kachuee, M., Fazeli, S., and Sarrafzadeh, M. (2018). "ECG heartbeat classification: a deep transferable representation," in Proceedings of the IEEE International Conference on Healthcare Informatics, (New York, NY: Institute of Electrical and Electronics Engineers). doi: 10.3390/s21217233

Kiranyaz, S., Ince, T., and Gabbouj, M. (2016). "Real-time patient-specific ECG Classification by 1-D convolutional neural networks," in Proceedings of the IEEE Transactions on Biomedical Engineering, (Piscataway, NJ: Institute of Electrical and Electronics Engineers), 664-675. doi: 10.1109/TBME.2015.2468589

Lapique, L. (1907). Recherches quantitatives sur l'excitation electrique des nerfs traitee comme une polarization. J. Physiol. Pathol. 9, 620-635.

Mehra, M. R., Desai, S. S., Kuy, S. R., Henry, T. D., and Patel, A. N. (2020). Cardiovascular disease, drug therapy, and mortality in Covid-19. N. Engl. J. Med. 382:e102.

Milo, V. (2020). "Modeling and simulation of spiking neural networks with resistive switching synapses," in Special Topics in Information Technology. SpringerBriefs in Applied Sciences and Technology, ed. B. Pernici (Cham: Springer). doi: 10.1007/978-3-030-32094-2_4

Ozkan, H., Ozhan, O., Karadana, Y., Gulcu, M., Macit, S., Husain, F., et al. (2019). "A portable wearable tele-ECG monitoring system," in Proceedings of the IEEE Transactions on instrumentation and measurement, (Piscataway, NJ: Institute of Electrical and Electronics Engineers), 173-182. doi: 10.1109/TIM.2019.2895484

Patan, R., Ghantasala, G. P., Sekaran, R., Gupta, D., and Ramachandran, M. (2020). Smart healthcare and quality of service in IoT using grey filter convolutional based cyber physical system. Sustain. Cities and Soc. 59:102141. doi: 10.1016/j. scs.2020.102141

Peng, X., Huang, S., Jiang, H., Lu, A., and Yu, S. (2020). "DNN+NeuroSim V2.0: an end-to-end benchmarking framework for compute-in-memory accelerators for on-chip training," in Proceedings of the IEEE Transactions on Computer-Aided Design of Integrated Circuits and Systems, (Piscataway, NJ: Institute of Electrical and Electronics Engineers ), doi: 10.1109/TCAD.2020.3043731

Ponulak, F., and Kasiński, A. (2010). Supervised learning in spiking neural networks with ReSuMe: sequence learning, classification, and spike shifting. Neural Comput. 22, 467-510. doi: 10.1162/neco.2009.11-08-901

$\mathrm{Pu}, \mathrm{D}$., and Cook, M. (2015). Unsupervised learning of digit recognition using spike-timing-dependent plasticity. Front. Comput. Neurosci. 9:99. doi: 10.3389/ fncom.2015.00099

Purushothaman, S., Salmani, D., Prarthana, K. G., Bandelkar, S. M., and Varghese, S. (2014). Study of ECG changes and its relation to mortality in cases of cerebrovascular accidents. J. Nat. Sci. Biol. Med. 5:434. doi: 10.4103/0976-9668. 136225

Pustokhina, I. V., Pustokhin, D. A., Gupta, D., Khanna, A., Shankar, K., and Nguyen, G. N. (2020). An effective training scheme for deep neural network in edge computing enabled Internet of medical things (IoMT) systems. IEEE Access 8, 107112-107123. doi: 10.1109/ACCESS.2020.3000322

Rajpurkar, P., Hannun, A. Y., Haghpanahi, M., Bourn, C., and Ng, A. Y. (2017). Cardiologist-level arrhythmia detection with convolutional neural networks. arXiv [Preprint] arXiv:1707.01836v1,

Saadatnejad, S., Oveisi, M., and Hashemi, M. (2020). "LSTM-based ECG classification for continuous monitoring on personal wearable devices," in Proceedings of the IEEE Journal of Biomedical and Health Informatics, (Piscataway, NJ: Institute of Electrical and Electronics Engineers), 515-523. doi: 10.1109/JBHI.2019.2911367

Shafiee, A., Nag, A., Muralimanohar, N., Balasubramonian, R., Strachan, J. P., Miao, H., et al. (2016). "ISAAC: a convolutional neural network accelerator with in-situ analog arithmetic in crossbars," in Proceedings of the 2016 ACM/IEEE 
43rd Annual International Symposium on Computer Architecture (ISCA), (IEEE: Seoul).

Sodhro, A. H., Pirbhulal, S., and Sangaiah, A. K. (2018). Convergence of iot and product lifecycle management in medical health care. Future Gener. Comput. Syst. 86, 380-391. doi: 10.1016/j.future.2018.03.052

Strukov, D. B., Snider, G. S., Stewart, D. R., and Williams, R. S. (2009). The missing memristor found. Nature 459, 1154-1154. doi: 10.1038/nature06932

Tang, T., Xia, L., Li, B., Rong, L., and Yang, H. (2015). "Spiking neural network with rram: can we use it for real-world application?", in Proceedings of the Design, Automation and Test in Europe, (Grenoble: Institute of Electrical and Electronics Engineers). doi: 10.7873/DATE.2015.1085

Tian, F., Yang, J., Zhao, S., and Sawan, M. (2021). "A new neuromorphic computing approach for epileptic seizure prediction," in Proceedings of the IEEE International Symposium on Circuits and Systems (ISCAS), (Daegu: Institute of Electrical and Electronics Engineers), 1-5. doi: 10.1186/s12868-0160283-6

Vafaie, M. H., Ataei, M., and Koofigar, H. R. (2014). Heart diseases prediction based on ECG signals' classification using a genetic-fuzzy system and dynamical model of ECG signals. Biomed. Signal Process. Control 14, 291-296. doi: 10. 1016/j.bspc.2014.08.010

Valentian, A., Rummens, F., Vianello, E., Mesquida, T., and Reita, C. (2019). "Fully integrated spiking neural network with analog neurons and RRAM synapses," in Proceedings of the IEEE International Electron Devices Meeting (IEDM), (San Francisco, CA: Institute of Electrical and Electronics Engineers).

Verma, N., Jia, H., Valavi, H., Tang, Y., and Deaville, P. (2019). In-memory computing: advances and prospects. IEEE Solid State Cir. Mag. 11, 43-55. doi: 10.1109/MSSC.2019.2922889

Wang, Z., Joshi, S., Savel'ev, S., Song, W., Midya, R., Li, Y., et al. (2018). Fully memristive neural networks for pattern classification with unsupervised learning. Nat. Electron. 1, 137-145. doi: 10.1038/s41928-018-0023-2

Wang, Z., Joshi, S., Savel'ev, S. E., Jiang, H., Midya, R., Lin, P., et al. (2017). Memristors with diffusive dynamics as synaptic emulators for neuromorphic computing. Nat. Mater. 16, 101-108. doi: 10.1038/nmat4756

Wilkins, E., Wilson, L., Wickramasinghe, K., Bhatnagar, P., Leal, J., LuengoFernandez, R., et al. (2017). European Cardiovascular Disease Statistics 2017. Brussels: European Heart Network.

Wu, Y., Deng, L., Li, G., Zhu, J., and Shi, L. (2018). Spatio-temporal backpropagation for training high-performance spiking neural networks. Front. Neurosci. 12:331. doi: 10.3389/fnins.2018.00331

Wu, Y., Liu, Y. H., Liu, S., Yu, Q., Chen, T. P., and Liu, Y. (2020). Spike-driven gated recurrent neural network processor for electrocardio-gram arrhythmias detection realised in 55-nm CMOS technology. Electron. Lett. 56, 1230-1232. doi: 10.1049/el.2020.2224

Xiao, T. P., Bennett, C. H., Feinberg, B., Agarwal, S., and Marinella, M. J. (2020). Analog architectures for neural network acceleration based on non-volatile memory. Appl. Phys. Rev. 7:031301. doi: 10.1088/1361-6528/ab47bc
Yang, Z., Huang, Y., Zhu, J., and Ye, T. T. (2020). “Analog circuit implementation of LIF and STDP models for spiking neural networks," in Proceedings of the 2020 on Great Lakes Symposium on VLSI, (New York, NY: Association for Computing Machinery), 469-474. doi: 10.3390/s21041065

Yasin, M., Tekeste, T., Saleh, H., Mohammad, B., Sinanoglu, O., and Ismail, M. (2017). "Ultra-low power, secure IoT platform for predicting cardiovascular diseases," in Proceedings of the IEEE Transactions on Circuits and Systems I: Regular Papers, (Piscataway, NJ: Institute of Electrical and Electronics Engineers), 2624-2637. doi: 10.1109/tcsi.2017.26 94968

Zhang, W., Yu, L., Ye, L., Zhuang, W., and Ma, F. (2018). "ECG signal classification with deep learning for heart disease identification," in Proceedings of the International Conference on Big Data and Artificial Intelligence, (Beijing: Institute of Electrical and Electronics Engineers).

Zhang, X., Wu, Z., Lu, J., Wei, J., and Liu, M. (2020). "Fully memristive SNNs with temporal coding for fast and low-power edge computing," in Proceedings of the IEEE International Electron Devices Meeting (IEDM), (San Francisco, CA: Institute of Electrical and Electronics Engineers). doi: 10.1109/IEDM13553. 2020.9371937

Zhang, Y., Wu, H., Qian, H., Kang, J., and Gao, B. (2019). “An improved rram-based binarized neural network with high variation-tolerated forward/backward propagation module," in Proceedings of the IEEE Transactions on Electron Devices, (Piscataway, NJ: Institute of Electrical and Electronics Engineers), 1-5.

Zhou, D., Xi, B., Zhao, M., Wang, L., and Veeranki, S. P. (2018). Uncontrolled hypertension increases risk of all-cause and cardiovascular disease mortality in US adults: the NHANES III linked mortality study. Sci. Rep. 8:9418. doi: 10.1038/s41598-018-27377-2

Conflict of Interest: The authors declare that the research was conducted in the absence of any commercial or financial relationships that could be construed as a potential conflict of interest.

Publisher's Note: All claims expressed in this article are solely those of the authors and do not necessarily represent those of their affiliated organizations, or those of the publisher, the editors and the reviewers. Any product that may be evaluated in this article, or claim that may be made by its manufacturer, is not guaranteed or endorsed by the publisher.

Copyright (c) 2021 Jiang, Tian, Liang, Shen, Liu, Zheng, Wu, Zhang, Fang, Zhao, Shi, Xue and Zeng. This is an open-access article distributed under the terms of the Creative Commons Attribution License (CC BY). The use, distribution or reproduction in other forums is permitted, provided the original author(s) and the copyright owner(s) are credited and that the original publication in this journal is cited, in accordance with accepted academic practice. No use, distribution or reproduction is permitted which does not comply with these terms. 